\title{
The impact of the duration of an untreated episode on improvement of depression and somatic symptoms
}

This article was published in the following Dove Press journal:

Neuropsychiatric Disease and Treatment

27 August 2015

Number of times this article has been viewed

\author{
Ching-I Hung ${ }^{1,2}$ \\ Nan-Wen Yu ${ }^{1,2}$ \\ Chia-Yih Liu' ${ }^{1,2}$ \\ Kuan-Yi Wu ${ }^{1,2}$ \\ Ching-Hui Yang ${ }^{3}$ \\ 'Department of Psychiatry, Chang \\ Gung Memorial Hospital, Linkou, \\ Taiwan, ${ }^{2}$ Chang Gung University \\ College of Medicine, Tao-Yuan, Taiwan; \\ ${ }^{3}$ Department of Nursing, Chang Gung \\ University of Science and Technology, \\ Tao-Yuan, Taiwan
}

Purpose: The aim of this study was to investigate the impact of the duration of an untreated episode (DUE) on the improvement of depression and somatic symptoms among patients with major depressive disorder (MDD), after the patients had received 4 weeks of pharmacotherapy.

Methods: In this open-label study, there were 155 participants with MDD who were treated daily with $75 \mathrm{mg}$ of venlafaxine for 4 weeks. DUE was defined as the interval between the onset of the index major depressive episode and the start of pharmacotherapy. The Depression and Somatic Symptoms Scale (DSSS), composed of the depression subscale (DS) and the somatic subscale (SS), was used. The SS included the pain subscale (PS) and the nonpain somatic subscale (NPSS). Multiple linear regressions were used to test the impacts of DUE on the improvement percentages (IPs) of depression and somatic symptoms.

Results: Eighty-five subjects completed the 4-week treatment. The IPs of the DS, SS, and NPSS were significantly negatively correlated with DUE. A shorter DUE was related to higher IPs. DUE was an independent factor, predicting the IPs of the DS, SS, and NPSS. DUE $<1$ month was the most powerful time-point to predict the IPs of the DS, SS, and NPSS. However, DUE was unable to predict the IP of the PS at all time-points.

Conclusion: A shorter DUE might be one of the factors related to greater improvement of depression and somatic symptoms. DUE should be considered as an important factor when investigating the prognosis of depression and somatic symptoms.

Keywords: early intervention, somatization, treatment response, prognosis, outcome

\section{Introduction}

Many studies have investigated the treatment response and prognosis of depressive disorders post-pharmacotherapy. The duration of untreated illness (DUI) and duration of an untreated episode (DUE) are important factors related to the prognosis of major depressive disorder (MDD). ${ }^{1-7}$ The remission rate of patients with MDD gradually decreases with a longer DUI. ${ }^{3}$ Response to antidepressant treatments is faster when DUE is reduced among patients with MDD. ${ }^{4}$ The remission rate was significantly decreased among depressive patients with DUI $\geq 6$ months as compared to patients who were treated with pharmacotherapy earlier after onset, and the negative influence of a prolonged DUI on the outcome of depression did not seem to be confounded by demographic and clinical variables. ${ }^{2}$

The severity of somatic symptoms is correlated with that of depression. ${ }^{8}$ Somatic symptoms are common residual symptoms among patients with MDD. ${ }^{9}$ Somatic symptom severity at baseline is one of the important factors related to the prognosis of depression. ${ }^{10,11}$ Increased severity of somatic symptoms is associated with decreased treatment response and remission rates. ${ }^{8}$ Painful physical symptoms (PPS) at baseline
Correspondence: Ching-I Hung Department of Psychiatry, Chang Gung Memorial Hospital, 5 Fu-Shing Street, Linkou, Kweishan, Taoyuan 333, Taiwan Tel +886 3328 I 200 ext 2439

Fax +88633280267

Email a36250@cgmh.org.tw 
predict a poorer quality of life posttreatment. ${ }^{12}$ Therefore, somatic symptoms and PPS have negative impacts on the prognosis of MDD.

Although there have been studies investigating the impacts of DUI and DUE on the prognosis of MDD, these studies have focused on the treatment response or remission of depression. ${ }^{1}$ To the best of our knowledge, no study has investigated the impacts of DUE on the improvement of somatic symptoms post-pharmacotherapy among patients with MDD. ${ }^{1}$ Clarification of this issue is important because somatic symptoms have negative impacts on the prognosis of MDD. Therefore, the purpose of this study was to investigate the impact of DUE on the improvement of depression and somatic symptoms after 4 weeks of pharmacotherapy. We hypothesized that patients with a shorter DUE would show greater improvement of somatic symptoms after treatment.

\section{Methods}

\section{Subjects}

This study was conducted in the psychiatric outpatient clinics of the Chang Gung Memorial Hospital, a medical center in Northern Taiwan, from September 2005-August 2007. The study was approved by the Institutional Review Board of the Chang Gung Memorial Hospital.

Subjects between 18 and 65 years of age were recruited from a group of consecutive outpatients. The subjects had not taken antidepressants or other psychotropic drugs within the previous 4 weeks. Patients who met the $D S M-I V$-text revision (TR) criteria for MDD and were currently experiencing a major depressive episode (MDE) were enrolled. ${ }^{13} \mathrm{MDD}$ and anxiety disorders were diagnosed using the Structured Clinical Interview for DSM-IV-TR Axis I Disorders. ${ }^{14}$ To prevent depressive and somatic symptoms from being confounded, three exclusion criteria were established: 1) psychotic symptoms, severe psychomotor retardation, or catatonic features; 2) a history of substance dependence or abuse without complete remission in the past month; and 3) chronic medical diseases such as diabetes mellitus, hypertension, and other medical diseases. Written informed consent was obtained from all patients prior to their entrance into the study. To decrease the rejection rate due to unknown medication efficacies and side effects, the study was designed as an open-label study.

All subjects were informed of three issues: 1) antidepressants used in the project had previously been proven to be effective for the treatment of MDD and, therefore, this study was not a trial to establish the efficacy of a new antidepressant or to develop a new indication; 2) the period with a fixeddosage antidepressant was only 4 weeks, after which, there would be no limitations in terms of medications; 3 ) subjects were educated regarding depression and treatment issues. An information sheet was provided to all subjects.

\section{Psychometric scales}

The DSSS and the 17-item Hamilton Depression Rating Scale (HAMD) were used. ${ }^{15-18}$ The DSSS includes twelve items of the DS and ten items of the SS. As the HAMD was not designed for the evaluation of somatic symptoms, the DSSS was used as the main scale to evaluate depression and somatic symptoms. Among the ten items of the SS, five items were for pain symptoms (headache, back pain, chest pain, neck or shoulder soreness, and muscle soreness), which constituted the pain subscale (PS); five items were for nonpain somatic symptoms (chest tightness, muscle tension, dizziness, shortness of breath, and palpitation), which constituted the nonpain somatic subscale (NPSS). In developing the DSSS, the ten items of the SS were selected based on: 1) somatic items that could reflect the severity of depression, predict the prognosis of depression, or have significant impacts on clinical practice; and 2) somatic symptoms that were common in previous studies for depression. The DSSS and its subscales are sensitive to pharmacotherapy and are significantly correlated with the HAMD. ${ }^{16}$ The DS and SS are significantly correlated with the mental and physical subscale scores of a health-related quality of life scale, respectively. ${ }^{17}$ The predictive ability of the DSSS for the prognosis of depression is not inferior to that of the HAMD. ${ }^{10}$ The total scores for each subscale ranged from 0-36 for the DS, 0-30 for the SS, and 0-15 for the PS and the NPSS. A higher score indicated a greater severity.

\section{Procedure}

DUE was defined as the interval between the onset of the current MDE and the start of pharmacotherapy, in this study. A board-certified psychiatrist, who was blind to other data, interviewed these patients to clarify the onset time-point of the index MDE, based on the Structured Clinical Interview for DSM-IV-TR Axis I Disorders. ${ }^{14}$ Then, the interval between the onset of the current MDE and the start of pharmacotherapy was calculated.

The patients were treated for 4 weeks with venlafaxine extended-release, ie, one $75 \mathrm{mg}$ capsule per day. Drug compliance was confirmed by the method of pill counting. Zolpidem (10 mg per tablet) was prescribed as needed only in the first 2 weeks, with a total amount of six tablets or fewer, because insomnia is common among patients with MDD. Four weeks later, the DSSS was applied. The HAMD was reevaluated by the same psychiatrist. 


\section{Statistical methods}

All statistical analyses were performed using SPSS for Windows 12.0 (SPSS Inc., IBM Corporation, Armonk, NY, USA). As there was no clear definition of the remission of somatic symptoms, the improvement percentage (IP), which was calculated as (score at baseline - score after 4-week treatment)/score at baseline, was used as the main indicator of prognosis. Only subjects with a venlafaxine treatment compliance of $\geq 80 \%$ (calculated as total capsules/total days) were included for further analyses. The independent $t$-test, paired $t$-test, and Pearson's correlation were used appropriately. Bonferroni corrections were used in the independent $t$-tests with multiple comparisons.

To understand the impacts of DUE on IP of depression and somatic symptoms after controlling for the severity of depression at baseline and demographic variables, two multiple linear regressions with forward selection were used. The first regression model tested whether DUE was an independent factor to predict improvement of depression and somatic symptoms after controlling for confounding factors. The second regression model tested each time-point of DUE in the first 6 months to determine which time-point was the most powerful for predicting the improvement of depression and somatic symptoms. The dependent variable was the IP of the depression and somatic scores in both models.

In the first regression model, the independent variables were demographic variables (sex, age, marital status, employment status, and number of years of education), DUE, presence or absence of anxiety comorbidities, age at the first MDE, whether or not it was the first MDE, and the
HAMD score at baseline. In the second regression model, the independent variables were the same as those in the first regression model, except that DUE was split into six categories, which were DUE $<1$ month (yes or no), DUE $<2$ months, DUE $<3$ months, DUE $<4$ months, DUE $<5$ months, and DUE $<6$ months. A two-tailed $P$-value $<0.05$ was considered statistically significant in all statistical analyses.

\section{Results \\ Subjects}

During the study period, 155 subjects agreed to participate in the study. Table 1 shows the demographic variables of the patients. Among them, $85(54.8 \%)$ patients with good compliance ( $\geq 80 \%$ compliance) were included in the further analyses (treatment group). The mean amount of zolpidem was 2.1 \pm 2.4 (mean $\pm \mathrm{SD}$ ) tablets during the first 2-week treatment period. None of the 85 patients had been treated by pharmacotherapy or psychotherapy for the current MDE before their entrance into the study. The other 70 patients comprised the withdrawal group, and included 22 patients who finished the 4 weeks of treatment but had poor compliance or were shifted to other antidepressants, and 48 patients who did not finish the 4 weeks of treatment. No significant differences were noted in the demographic variables and the depression and somatic severities between the treatment and withdrawal groups (Table 1). The withdrawal group had a shorter DUE as compared with the treatment group $(P=0.07)$.

Among the 85 patients, 43 patients $(50.6 \%)$ had at least one of the following anxiety comorbidities: $10.6 \%(n=9)$ panic disorder and/or agoraphobia, 25.9\% $(n=22)$ social

Table I Demographic variables and mean scores of psychometric scales at baseline

\begin{tabular}{llll}
\hline Demographic variable & $\begin{array}{l}\text { Total sample } \\
(\mathbf{N}=\mathbf{1 5 5 )}\end{array}$ & $\begin{array}{l}\text { Treatment group } \\
(\mathbf{N}=\mathbf{8 5})\end{array}$ & $\begin{array}{l}\text { Withdrawal group } \\
(\mathbf{N}=\mathbf{7 0})\end{array}$ \\
\hline Age (years) & $30.3 \pm 8.0$ & $29.5 \pm 7.3$ & $31.2 \pm 8.7$ \\
Number of years of education & $13.4 \pm 2.5$ & $13.6 \pm 2.7$ & $13.1 \pm 2.2$ \\
Sex (female \%) & 68.4 & 63.5 & 74.3 \\
Married (\%) & 38.7 & 38.8 & 38.6 \\
Employed (\%) & 59.4 & 56.5 & 62.9 \\
Age at first episode (years) & $27.4 \pm 8.5$ & $26.3 \pm 7.5$ & $28.6 \pm 9.4$ \\
First major depressive episode (yes \%) & 76.1 & 74.1 & 78.6 \\
Duration of an untreated episode (months) & $16.9 \pm 24.0$ & $19.9 \pm 27.3$ & $13.2 \pm 18.9$ \\
HAMD score & $23.4 \pm 4.0$ & $23.2 \pm 3.9$ & $23.6 \pm 4.1$ \\
DS score & $25.6 \pm 5.4$ & $25.3 \pm 5.7$ & $25.9 \pm 5.1$ \\
SS score & $16.1 \pm 6.6$ & $16.3 \pm 6.7$ & $15.9 \pm 6.6$ \\
PS score & $7.6 \pm 3.8$ & $7.9 \pm 3.7$ & $7.2 \pm 3.9$ \\
NPSS score & $8.6 \pm 3.4$ & $8.5 \pm 3.5$ & $8.7 \pm 3.3$ \\
\hline
\end{tabular}

Notes: Data presented as mean \pm standard deviation or percentage. There were no significant differences in these demographic variables or severities of depression and somatic symptoms between the treatment and withdrawal groups.

Abbreviations: HAMD, Hamilton Depression Rating Scale; DSSS, Depression and Somatic Symptoms Scale; DS, depression subscale of the DSSS; SS, somatic subscale of the DSSS; PS, pain subscale of the DSSS; NPSS, nonpain somatic subscale of the DSSS. 
phobia, 22.4\% $(\mathrm{n}=19)$ specific phobia, $11.8 \%(\mathrm{n}=10)$ posttraumatic stress disorder, $9.4 \%(\mathrm{n}=8)$ obsessive-compulsive disorder, and $7.1 \%(\mathrm{n}=5)$ generalized anxiety disorder.

\section{The IPs of the scales and subscales between groups}

After treatment, the scores of the two scales were significantly (all $P<0.001$ ) decreased (scores at baseline and posttreatment, respectively, mean \pm SD: HAMD, 23.2 \pm 3.9 and $12.8 \pm 6.5$; DS, 25.3 \pm 5.7 and 13.9 \pm 8.0 ; SS, $16.3 \pm 6.7$ and $8.3 \pm 6.8$; PS, 7.9 \pm 3.7 and $3.9 \pm 3.5$; NPSS, $8.5 \pm 3.5$ and $4.4 \pm 3.6$ ). The IPs of the two scales are shown in Table 2. There were no significant differences in IPs between groups.

\section{IPs in different DUEs}

Table 3 shows the IPs in subjects with different categorical DUEs in the first 6 months. Significant differences between groups in the first 6 months (except for DUE $<2$ months) were noted in the IPs of the HAMD, DS, SS, and NPSS. The difference in the IP of the PS between groups was not significant.

\section{Correlation of the IPs with DUE and psychometric scores}

In the 85 subjects, the IPs of the DS, SS, and NPSS were significantly negatively correlated with DUE, with correlation coefficients of $r=-0.29(P<0.01), r=-0.25(P=0.02)$, and $r=-0.23(P=0.04)$, respectively. The correlations of the IPs of the HAMD $(r=-0.17, P=0.12)$ and PS $(r=-0.19, P=0.08)$ with DUE were not significant.

Among patients with DUE $<24$ months ( $\mathrm{n}=58$ ), the IPs of the HAMD $(r=-0.38, P<0.01)$, DS $(r=-0.27, P=0.045)$, and NPSS $(r=-0.32, P=0.02)$ were significantly correlated with DUE; the correlations of DUE with the IPs of the SS $(r=-0.25, P=0.055)$ and PS $(r=-0.09, P=0.54)$ were borderline significant and insignificant, respectively. Among patients with DUE $\geq 24$ months $(n=27)$, the correlations of DUE with the IPs of the HAMD ( $r=-0.25)$, DS $(r=-0.30)$, SS $(r=-0.20)$, NPSS $(r=-0.18)$, and PS $(r=-0.19)$ were insignificant $(P>0.05)$.

The IPs of the HAMD, DS, SS, PS, and NPSS were not correlated with the psychometric scores at baseline, except for the IP of the HAMD to the SS score $(r=-0.27$, $P=0.01)$, PS score $(r=-0.22, P<0.05)$, and NPSS score $(r=-0.29, P<0.01)$ at baseline. The IPs of the HAMD, DS, SS, PS, and NPSS were not correlated with present age, number of years of education, or onset age of the first MDE.

\section{Factors independently predicting the IPs of somatic symptoms}

Table 4 shows the factors that independently predicted the IPs of depression and somatic symptoms. In the first model, DUE independently predicted the IPs of the DS, SS, and NPSS. Although DUE was not a significant factor related to the IP of the HAMD in the full sample ( $n=85)$, DUE was a significant factor related to the IP of the HAMD ( $\beta$-value $=-0.38$, adjusted $\left.R^{2}=0.13, P<0.01\right)$ among patients with DUE $<24$ months $(\mathrm{n}=58)$. In the second model, DUE $<1$ month was the only significant factor to predict the IPs of the DS, SS, and NPSS. DUE $<4$ months predicted the IP of the HAMD. DUE did not predict the IPs of PS in the two regression models. Demographic variables, the HAMD scores at baseline, age of the first episode, presence of anxiety comorbidities, and whether or not it was the first MDE, did not enter into the regression model.

Table 2 Improvement percentages of the two scales and subscales in groups after 4 weeks of treatment

\begin{tabular}{|c|c|c|c|c|c|c|}
\hline IPs in different groups & Number & HAMD & DS & SS & NPSS & PS \\
\hline IP for the full treatment group & 85 & $45.2 \pm 26.2$ & $44.6 \pm 29.9$ & $49.4 \pm 36.5$ & $48.2 \pm 36.6$ & $46.9 \pm 44.9$ \\
\hline IP in patients with anxiety comorbidity & 43 & $45.0 \pm 25.8$ & $42.1 \pm 30.2$ & $45.1 \pm 33.2$ & $43.9 \pm 33.6$ & $44.1 \pm 38.7$ \\
\hline IP in patients without anxiety comorbidity & 42 & $45.4 \pm 26.9$ & $47.1 \pm 29.7$ & $53.8 \pm 39.5$ & $52.9 \pm 39.3$ & $50.0 \pm 51.4$ \\
\hline IP in patients experiencing a first episode & 63 & $45.9 \pm 27.2$ & $44.4 \pm 31.1$ & $47.3 \pm 38.2$ & $47.3 \pm 38.4$ & $42.1 \pm 47.8$ \\
\hline IP in patients with multiple episodes & 22 & $43.4 \pm 23.7$ & $45.0 \pm 26.8$ & $55.5 \pm 31.0$ & $50.9 \pm 31.5$ & $59.7 \pm 33.6$ \\
\hline IP in male patients & 31 & $50.9 \pm 25.7$ & $48.3 \pm 25.1$ & $50.2 \pm 39.2$ & $48.0 \pm 39.2$ & $45.7 \pm 53.0$ \\
\hline IP in female patients & 54 & $42.0 \pm 26.2$ & $42.4 \pm 32.3$ & $48.9 \pm 35.2$ & $48.4 \pm 35.4$ & $47.5 \pm 40.2$ \\
\hline IP in patients currently employed & 48 & $47.1 \pm 27.2$ & $48.1 \pm 27.8$ & $52.1 \pm 35.9$ & $51.0 \pm 34.2$ & $50.7 \pm 47.8$ \\
\hline IP in patients currently unemployed & 37 & $42.8 \pm 25.1$ & $40.0 \pm 32.2$ & $45.8 \pm 37.4$ & $44.6 \pm 39.7$ & $41.9 \pm 40.9$ \\
\hline IP in married patients & 33 & $48.5 \pm 25.7$ & $46.5 \pm 26.5$ & $53.2 \pm 34.6$ & $49.7 \pm 31.7$ & $53.4 \pm 44.3$ \\
\hline IP in unmarried patients & 52 & $43.2 \pm 26.6$ & $43.3 \pm 31.4$ & $46.9 \pm 37.8$ & $47.3 \pm 39.7$ & $42.6 \pm 45.2$ \\
\hline
\end{tabular}

Note: Data presented as mean \pm standard deviation.

Abbreviations: IP, improvement percentage; HAMD, Hamilton Depression Rating Scale; DSSS, Depression and Somatic Symptoms Scale; DS, depression subscale of the DSSS; SS, somatic subscale of the DSSS; PS, pain subscale of the DSSS; NPSS, nonpain somatic subscale of the DSSS. 
Table 3 The improvement percentage at different durations of an untreated episode

\begin{tabular}{|c|c|c|c|c|c|c|}
\hline Duration of untreated episode & Number & HAMD & DS & SS & NPSS & PS \\
\hline \multicolumn{7}{|l|}{$<\mathrm{I}$ month } \\
\hline Yes & 9 & $63.9 \pm 21.1^{* \# \#}$ & $72.7 \pm 23.7$ *\#\# & $81.1 \pm 32.5^{* *}$ & $84.9 \pm 32.5 * *$ & $69.8 \pm 35.6$ \\
\hline No & 76 & $43.0 \pm 26.0$ & $41.2 \pm 28.9$ & $45.6 \pm 32.3$ & $43.9 \pm 34.7$ & $44.4 \pm 45.3$ \\
\hline \multicolumn{7}{|l|}{$<2$ months } \\
\hline Yes & 24 & $51.5 \pm 24.2$ & $57.8 \pm 22.4^{* \# \#}$ & $60.3 \pm 39.1$ & $60.4 \pm 36.9$ & $49.3 \pm 53.0$ \\
\hline No & 61 & $42.7 \pm 26.8$ & $39.3 \pm 31.0$ & $45.1 \pm 34.8$ & $43.4 \pm 35.6$ & $46.0 \pm 42.2$ \\
\hline \multicolumn{7}{|l|}{$<3$ months } \\
\hline Yes & 30 & $53.9 \pm 22.9$ *\# & $57.2 \pm 22.8^{* \# \#}$ & $61.2 \pm 35.4 *$ & $60.3 \pm 33.8^{*}$ & $54.2 \pm 48.2$ \\
\hline No & 55 & $40.5 \pm 26.9$ & $37.7 \pm 31.2$ & $42.9 \pm 35.8$ & $41.9 \pm 36.6$ & $43.2 \pm 43.2$ \\
\hline \multicolumn{7}{|l|}{$<4$ months } \\
\hline Yes & 36 & $53.1 \pm 21.7^{*, \#}$ & $55.3 \pm 21.3^{* \# \#}$ & $61.4 \pm 33.5 * *$ & $60.4 \pm 32.2 * *$ & $57.5 \pm 45.6$ \\
\hline No & 49 & $39.5 \pm 27.9$ & $36.7 \pm 32.9$ & $40.6 \pm 36.4$ & $39.6 \pm 37.3$ & $39.9 \pm 43.5$ \\
\hline \multicolumn{7}{|l|}{$<5$ months } \\
\hline Yes & 37 & $52.7 \pm 21.5^{\text {*\#\# }}$ & $54.8 \pm 21.2^{* \# \#}$ & $61.0 \pm 33.1 * *$ & $59.3 \pm 32.4^{* *}$ & $57.9 \pm 44.9$ \\
\hline No & 48 & $39.4 \pm 28.2$ & $36.6 \pm 33.2$ & $40.5 \pm 36.8$ & $40.0 \pm 37.6$ & $39.3 \pm 43.7$ \\
\hline \multicolumn{7}{|l|}{$<6$ months } \\
\hline Yes & $4 I$ & $52.0 \pm 23.6$ *\#\# & $53.8 \pm 24.0$ *\# & $60.0 \pm 34.1 * *$ & $58.9 \pm 32.5 * *$ & $55.6 \pm 47.8$ \\
\hline No & 44 & $38.9 \pm 27.2$ & $36.0 \pm 32.4$ & $39.5 \pm 36.2$ & $38.5 \pm 37.7$ & $39.5 \pm 41.4$ \\
\hline
\end{tabular}

Notes: Data presented as mean \pm standard deviation. $* P<0.05$. **Significance $(P<0.017)$ after Bonferroni corrections for independent $t$-test in SS, PS, and NPSS. ${ }^{*}$ Significance $(P<0.025)$ after Bonferroni corrections for independent $t$-test in the HAMD and DS.

Abbreviations: HAMD, Hamilton Depression Rating Scale; DSSS, Depression and Somatic Symptoms Scale; DS, depression subscale of the DSSS; SS, somatic subscale of the DSSS; PS, pain subscale of the DSSS; NPSS, nonpain somatic subscale of the DSSS.

\section{Discussion}

DUE was the most important factor, independently predicting the IPs of the DS, SS, and NPSS (Table 4). The IPs of the DS, SS, and NPSS were significantly negatively correlated with DUE. Therefore, a shorter DUE might be one of the factors related to a better IP of the DS and SS. However, the impacts of DUE on the improvement of somatic symptoms were specific to the NPSS. The correlation between DUE and the IP of the PS was not significant. Moreover, DUE was unable to predict the IP of the PS in the two regression models. These results have clinical implications. First, there is a possibility that earlier treatment with pharmacotherapy might help to improve both depression and nonpain somatic symptoms. Although this hypothesis needs further research, previous studies have demonstrated that a long duration without treatment has negative impacts on the course of MDD. ${ }^{1,6,7}$ Second, PPS and nonpain somatic symptoms might differ in terms of clinical characteristics. Novick et al reported that PPS was most associated with the prognosis of depression as compared with other somatic symptoms. ${ }^{8}$ Moreover, using a

Table 4 Independent variables predicting the improvement percentages after 4 weeks of treatment in patients experiencing a major depressive episode

\begin{tabular}{|c|c|c|c|c|c|c|}
\hline $\begin{array}{l}\text { IPs of different } \\
\text { scales }\end{array}$ & $\begin{array}{l}\text { Regression } \\
\text { model }\end{array}$ & $\begin{array}{l}\text { Independent } \\
\text { variable }\end{array}$ & $\beta$ & Adjusted $R^{2}$ & $t$ & $P$-value \\
\hline \multirow[t]{2}{*}{ IP of HAMD score } & I & None & NA & NA & NA & NA \\
\hline & II & DUE $<4$ months & 0.26 & 0.06 & 2.43 & 0.02 \\
\hline \multirow[t]{2}{*}{ IP of DS score } & I & DUE & -0.29 & 0.07 & -2.75 & $<0.01$ \\
\hline & II & DUE $<$ I month & 0.33 & 0.10 & 3.14 & $<0.01$ \\
\hline \multirow[t]{2}{*}{ IP of SS score } & I & DUE & -0.25 & 0.05 & -2.38 & 0.02 \\
\hline & II & DUE $<$ I month & 0.30 & 0.08 & 2.88 & $<0.01$ \\
\hline \multirow[t]{2}{*}{ IP of NPSS score } & I & DUE & -0.23 & 0.04 & -2.11 & 0.04 \\
\hline & II & DUE $<$ I month & 0.35 & 0.11 & 3.38 & $<0.01$ \\
\hline \multirow[t]{2}{*}{ IP of PS score } & I & None & NA & NA & NA & NA \\
\hline & II & None & NA & NA & NA & NA \\
\hline
\end{tabular}

Abbreviations: IP, improvement percentage; HAMD, Hamilton Depression Rating Scale; DS, depression subscale of the Depression and Somatic Symptoms Scale (DSSS); DUE, duration of an untreated episode; SS, somatic subscale of the DSSS; PS, pain subscale of the DSSS; NA, not applicable; NPSS, nonpain somatic subscale of the DSSS. 
low dose of venlafaxine and a short duration of treatment in the present study were also possible causes of the insignificant correlation of DUE with the IP of the PPS.

Bukh et al reported that patients with an untreated duration of depression $<6$ months had a higher remission rate than patients with an untreated duration $\geq 6$ months. ${ }^{2}$ Our study further investigated which time-point of DUE was the most powerful factor to predict IPs. In the second regression model, DUE $<1$ month was the most powerful factor to predict the IPs of the DS, SS, and NPSS. Among patients with DUE $<1$ month post 4-week pharmacotherapy, the IPs (Table 3) were 72.7 $\pm 23.7,81.1 \pm 32.5,69.8 \pm 35.6$, and 84.9 \pm 32.5 for DS, SS, PS, and NPSS, respectively. A good treatment response is important, because it might provide the confidence of patients to continue to accept pharmacotherapy and also encourage patients to seek treatment for future episodes. In fact, a poor response to pharmacotherapy is an important factor in dropping out from treatment. ${ }^{19}$

Several possible reasons might explain the good IPs with a shorter DUE. First, glucocorticoid levels are increased in MDD, and a prolonged high level may damage hippocampal neurons. ${ }^{20}$ Patients experiencing a current depressive episode have been found to have a reduction in hippocampal volume, and an increased length of depression has been associated with a smaller hippocampal volume. ${ }^{21,22}$ Antidepressants may upregulate neurogenesis. ${ }^{23}$ Therefore, early intervention by pharmacotherapy may prevent cerebral atrophy, which is probably reversible in the early stage of depression. Second, self-recovery without treatment has a higher possibility of occurring within the first 3 months of a depressive episode. ${ }^{24}$ Improvement of depression and somatic symptoms might result from a mixture of the effects of pharmacotherapy and spontaneous remission. The two effects were unable to be distinguished in this study. Third, those who seek treatment in an early stage of the onset of their depression might have a better mental health knowledge or social support system, which might encourage patients to seek help. In fact, a higher education level and income are related to a higher remission rate. ${ }^{25}$ Improvement of depression during an early stage also prevented patients' social support systems or financial conditions from collapsing (events which might exacerbate depression). Fourth, this study was an open-label study, and all patients understood that they were being treated with an antidepressant. This might have increased the expectation of improvement of depression and somatic symptoms. It is known that the placebo effect, which can influence patients' outcome, includes many factors. ${ }^{26}$ There is a possibility that the placebo effect is more significant in the early stage of depression because the damage to neurons may not be so severe and, therefore, neurons may recover more readily.

There are several points worth noting. First, the IPs of the DS, SS, and NPSS were significantly negatively correlated with DUE. This meant that a longer untreated duration was associated with a poorer treatment improvement. This result was compatible with previous studies. ${ }^{1-4}$ Second, a previous study has reported that a greater severity of depression at baseline is related to a poorer treatment response. ${ }^{27}$ In this study, the IPs of the DS, SS, and NPSS were correlated with DUE, but not with the severity of depression (HAMD) at baseline. Moreover, depressive severity at baseline was not a significant factor in the two regression models. This demonstrated that the impact of DUE on improvement of DS might be greater than the impact of the severity of depression at baseline. However, more evidence may be needed to support our results. Third, although DUE was not a significant factor related to the IP of the HAMD in the full sample (Table 4), DUE was a significant factor related to the IP of the HAMD among patients with DUE $<24$ months, and DUE $<4$ months in the second regression model. DUE was also a significant factor related to the IP of the DS, which correlated well with the HAMD. ${ }^{16}$ Our results only implied that DUE might be a possible factor related to the IP of depression. The role of DUE in the IP of depression should be further clarified.

There are several methodological issues or limitations in this study. First, this study had no placebo-controlled group because the study did not aim to test the efficacy of venlafaxine. Therefore, the IPs in this study do not represent the efficacy of venlafaxine. Second, there is no definition or criteria for somatic symptoms of MDD in the DSM-IV-TR criteria. Some somatic symptoms overlap with side effects of antidepressants, such as dry mouth, nausea, and gastrointestinal discomfort. Commonly-used somatic scales include different somatic items. More evidence is needed to support our results, which should be replicated by other somatic scales. Third, the treatment duration of this study was shorter than the treatment durations of previous studies, because 4-week treatment may be a milestone after which a treatment response can be observed. ${ }^{1,28-30}$ Fourth, this study did not separate patients experiencing a first MDE from those who had experienced multiple MDEs. To resolve this issue, whether or not a patient was experiencing their first MDE was used as an independent variable in the two regression models. The results showed that this variable was not significant. Fifth, insignificant correlations of DUE with the IPs of scales and subscales among patients with DUE $\geq 24$ months might result from following reasons: the sample size was small in the group; the course of depression might fluctuate; and if the DUE was too long, 
it might be difficult to clarify, and memory bias might exist. Finally, although zolpidem usage was limited in the first 2 weeks, and the total mean amount was only $2.1 \pm 2.4$ tablets, possible bias could not be excluded.

\section{Conclusion}

DUE is an important factor, predicting the IPs of the DS, SS, and NPSS in the regression models. DUE $<1$ month was the most powerful factor to predict the IPs of the DS, SS, and NPSS. DUE was negatively correlated with the IPs of the DS, SS, and NPSS. A shorter DUE might be one of the factors associated with better IPs. Future studies should consider DUE as an important factor for the treatment prognosis of depression and somatic symptoms among patients with MDD.

\section{Acknowledgment}

This study was supported in part by National Science Council grants NSC 94-2314-B-182A-207 and NSC 95-2314-B182A-188-MY2.

\section{Disclosure}

The authors report no conflicts of interest in this work.

\section{References}

1. Ghio L, Gotelli S, Marcenaro M, Amore M, Natta W. Duration of untreated illness and outcomes in unipolar depression: a systematic review and meta-analysis. J Affect Disord. 2014;152-154:45-51.

2. Bukh JD, Bock C, Vinberg M, Kessing LV. The effect of prolonged duration of untreated depression on antidepressant treatment outcome. $J$ Affect Disord. 2013;145(1):42-48.

3. Okuda A, Suzuki T, Kishi T, et al. Duration of untreated illness and antidepressant fluvoxamine response in major depressive disorder. Psychiatry Clin Neurosci. 2010;64(3):268-273.

4. de Diego-Adelino J, Portella MJ, Puigdemont D, Perez-Egea R, AlvarezE, Perez V. A short duration of untreated illness (DUI) improves response outcomes in first-depressive episodes. J Affect Disord. 2010;120(1-3): 221-225.

5. Altamura AC, Buoli M, Albano A, Dell'Osso B. Age at onset and latency to treatment (duration of untreated illness) in patients with mood and anxiety disorders: a naturalistic study. Int Clin Psychopharmacol. 2010;25(3):172-179.

6. Altamura A, Dell'osso B, Vismara S, Mundo E. May duration of untreated illness influence the long-term course of major depressive disorder? Eur Psychiatry. 2008;23(2):92-96.

7. Altamura AC, Dell'Osso B, Mundo E, Dell'Osso L. Duration of untreated illness in major depressive disorder: a naturalistic study. Int J Clin Pract. 2007;61(10):1697-1700.

8. Novick D, Montgomery W, Aguado J, et al. Which somatic symptoms are associated with an unfavorable course in Asian patients with major depressive disorder? J Affect Disord. 2013;149(1-3):182-188.

9. Hung CI, Liu CY, Wang SJ, Yang CH. Residual symptoms related to physical and panic symptoms at baseline predict remission of depression at follow-up. Psychopathology. 2014;47(1):51-56.

10. Hung CI, Liu CY, Wang SJ, Juang YY, Yang CH. Somatic symptoms: an important index in predicting the outcome of depression at six-month and two-year follow-up points among outpatients with major depressive disorder. J Affect Disord. 2010;125(1-3):134-140.
11. Köhler S, Unger T, Hoffmann S, Mackert A, Ross B, Fydrich T. The relationship of health-related quality of life and treatment outcome during inpatient treatment of depression. Qual Life Res. 2015;(3)24: 641-649.

12. Novick D, Montgomery W, Kadziola Z, et al. Do concomitant pain symptoms in patients with major depression affect quality of life even when taking into account baseline depression severity? Patient Prefer Adherence. 2013;7:463-470.

13. American Psychiatric Association. Diagnostic and Statistical Manual of Mental Disorders, Fourth Edition, Text Revision. Washington, DC: American Psychiatric Association; 2000.

14. First MB, Spitzer RL, Gibbon M, Williams JBW. Structured Clinical Interview for DSM-IV-TR Axis I Disorders, Research Version, Patient Edition (SCID-I/P). New York: Biometrics Research, New York State Psychiatric Institute; 2002.

15. Hamilton M. Development of a rating scale for primary depressive illness. Br J Soc Clin Psychol. 1967;6(4):278-296.

16. Hung CI, Weng LJ, Su YJ, Liu CY. Depression and Somatic Symptoms Scale: a new scale with both depression and somatic symptoms emphasized. Psychiatry Clin Neurosci. 2006;60(6):700-708.

17. Hung CI, Wang SJ, Liu CY. Validation of the Depression and Somatic Symptoms Scale (DSSS) by comparison with the Short Form 36 (SF-36) scale among psychiatric outpatients with major depressive disorder. Depress Anxiety. 2009;26(6):583-591.

18. Hung CI, Liu CY, Wang SJ, Yao YC, Yang CH. The cut-off points of the Depression and Somatic Symptoms Scale and the Hospital Anxiety and Depression Scale in detecting non-full remission and a current major depressive episode. Int J Psychiatry Clin Pract. 2012;16(1):33-40.

19. Hung CI, Wang SJ, Liu CY, Hsu SC, Yang CH. Comorbidities and factors related to discontinuation of pharmacotherapy among outpatients with major depressive disorder. Compr Psychiatry. 2011;52(4):370-377.

20. Sapolsky RM, Uno H, Rebert CS, Finch CE. Hippocampal damage associated with prolonged glucocorticoid exposure in primates. J Neurosci. 1990;10(9):2897-2902.

21. Kempton MJ, Salvador Z, Munafò MR, et al. Structural neuroimaging studies in major depressive disorder: meta-analysis and comparison with bipolar disorder. Arch Gen Psychiatry. 2011;68(7):675-690.

22. Videbech P, Ravnkilde B. Hippocampal volume and depression: a metaanalysis of MRI studies. Am J Psychiatry. 2004;161(11):1957-1966.

23. Malberg JE, Eisch AJ, Nestler EJ, Duman RS. Chronic antidepressant treatment increases neurogenesis in adult rat hippocampus. J Neurosci. 2000;20(24):9104-9110.

24. Posternak MA, Solomon DA, Leon AC, et al. The naturalistic course of unipolar major depression in the absence of somatic therapy. J Nerv Ment Dis. 2006;194(5):324-329.

25. Trivedi MH, Rush AJ, Wisniewski SR, et al. Evaluation of outcomes With citalopram for depression using measurement-based care in STAR*D: implications for clinical practice. Am J Psychiatry. 2006;163(1): $28-40$.

26. Preskorn, Sheldon $H$. What do the terms "drug-specific response/remission rate" and "placebo" really mean? J Psychiatry Pract. 2011;17(6): $420-424$.

27. Friedman ES, Davis LL, Zisook S, et al. Baseline depression severity as a predictor of single and combination antidepressant treatment outcome: results from the CO-MED trial. Eur Neuropsychopharmacol. 2012(3); 22:183-199.

28. Crismon ML, Trivedi M, Pigott TA, et al. The Texas Medication Algorithm Project: report of the Texas Consensus Conference Panel on Medication Treatment of Major Depressive Disorder. J Clin Psychiatry. 1999; 60(3):142-156.

29. Quitkin FM, McGrath PJ, Stewart JW, et al. Chronological milestones to guide drug change. When should clinicians switch antidepressants? Arch Gen Psychiatry. 1996;53(9):785-792.

30. Tedeschini E, Fava M, Papakostas GI. Placebo-controlled, antidepressant clinical trials cannot be shortened to less than 4 weeks' duration: a pooled analysis of randomized clinical trials employing a diagnostic odds ratio-based approach. J Clin Psychiatry. 2011;72(1):98-113. 


\section{Publish your work in this journal}

Neuropsychiatric Disease and Treatment is an international, peerreviewed journal of clinical therapeutics and pharmacology focusing on concise rapid reporting of clinical or pre-clinical studies on a range of neuropsychiatric and neurological disorders. This journal is indexed on PubMed Central, the 'PsycINFO' database and CAS,

and is the official journal of The International Neuropsychiatric Association (INA). The manuscript management system is completely online and includes a very quick and fair peer-review system, which is all easy to use. Visit http://www.dovepress.com/testimonials.php to read real quotes from published authors.

Submit your manuscript here: http://www.dovepress.com/neuropsychiatric-disease-and-treatment-journal 